\title{
COMPLICAÇÕES PÓS-OPERATÓRIAS EM APENDICECTOMIAS: ANÁLISE COMPARATIVA ENTRE AS ABORDAGENS ABERTA E VIDEOLAPAROSCÓPICA
}

\section{POSTOPERATIVE COMPLICATIONS IN APPENDECTOMIES: COMPARATIVE ANALYSIS BETWEEN OPEN AND LAPAROSCOPIC APPROACHES}

\author{
Mariana Kumaira Fonseca , Carlos Eduardo Bastian da Cunha', \\ Eduardo Zanotta Rodrigues ${ }^{1}$, Ricardo Breigeiron ${ }^{1}$, Miguel Prestes Nácul ${ }^{1}$
}

\section{RESUMO}

Clin Biomed Res. 2021;41(4):306-312

1 Serviço de Cirurgia Geral e do Trauma, Hospital de Pronto Socorro de Porto Alegre. Porto Alegre, RS, Brasil.

Autor correspondente: Mariana Kumaira Fonseca marianakumaira@gmail.com Hospital de Pronto Socorro de Porto Alegre

Largo Teodoro Herlz s/n 90040-192, Porto Alegre, RS, Brasil.
Introdução: A apendicectomia é o tratamento de escolha da apendicite aguda. Embora a preferência pelas técnicas minimamente invasivas seja tendência mundial, a cirurgia aberta ainda é realidade na maioria dos hospitais públicos. O índice de complicações pós-operatórias varia de acordo com a técnica cirúrgica empregada. O presente estudo objetiva comparar a incidência de complicações pós-operatórias entre a apendicectomia aberta e laparoscópica.

Métodos: Coorte retrospectiva incluindo pacientes submetidos à apendicectomia no Hospital de Pronto Socorro de Porto Alegre entre novembro de 2015 a novembro de 2019. Foram avaliados dados demográficos, tempo de evolução dos sintomas, técnica cirúrgica, achados transoperatórios, necessidade de drenos ou ostomias, tempo cirúrgico, tempo de internação, experiência do cirurgião e desfechos.

Resultados: Foram incluídos 358 pacientes, com idade de $32 \pm 13,8$ anos, e predomínio do sexo masculino (58,9\%); $58,1 \%$ foram submetidos a cirurgia aberta, $41,9 \%$ a laparoscopia e $8 \%$ necessitaram conversão. As apendicites foram classificadas como complicadas em um terço dos casos. O tempo cirúrgico foi menor na cirurgia aberta $(79,3 \pm 38,8$ vs. $104 \pm 35,2$ minutos; $p<0,001)$. O índice de complicações pós-operatórias foi de $21,2 \%$, sendo significativamente maior na técnica aberta ( $26,4 \%$ vs. $13 \% ; p=0,003)$. O tempo de internação, a necessidade de reintervenção e mortalidade não apresentaram diferença entre as técnicas.

Conclusão: Embora a apendicectomia aberta seja um procedimento seguro, com bons resultados e baixa morbimortalidade, a laparoscopia oferece potenciais vantagens em termos de evolução pós-operatória, inclusive em casos complicados. Deve ser indicada rotineiramente havendo disponibilidade de material e capacitação da equipe cirúrgica.

Palavras-chave: Apendicite; Apendicectomia; Complicações pós-operatórias

\section{ABSTRACT}

Introduction: Appendectomy is the treatment of choice for acute appendicitis. Although the preference for minimally invasive techniques is a worldwide trend, open surgery remains a reality in most public hospitals. The rate of postoperative complications varies according to the surgical technique employed. The present study aimed to compare the incidence of postoperative complications between open and laparoscopic appendectomy.

Methods: This retrospective cohort study included patients undergoing appendectomy at the Hospital de Pronto Socorro de Porto Alegre between November 2015 and November 2019. Demographic and clinical data, duration of symptoms, surgical 
technique, intraoperative findings, use of abdominal drains or stomas, operative time, length of stay, surgeon's experience, and outcomes were assessed.

Results: Three hundred and fifty-eight patients were included, predominantly male (58.9\%), with a mean age of $32 \pm 13.8$ years; $58.1 \%$ underwent open surgery, $41.9 \%$ underwent laparoscopic surgery, and $8 \%$ required conversion. One third of the cases were classified as complicated. The mean operative time was shorter for open surgery (79.3 \pm 38.8 vs. $104 \pm 35.2$ minutes; $p<0.001$ ). The rate of postoperative complications was $21.2 \%$, with a significantly higher incidence in the open technique $(26.4 \%$ vs. $13 \% ; p=0.003)$. Length of stay, reoperation rate, and mortality did not differ between the techniques.

Conclusions: Although open appendectomy is a safe and efficient procedure, associated with low morbidity and mortality rates, laparoscopy provides potential clinically beneficial advantages in terms of postoperative outcomes, even in complicated cases. Therefore, it should be routinely performed where laparoscopic equipment and skillful staff are available.

Keywords: Appendicitis; Appendectomy; Postoperative complications

\section{INTRODUÇÃO}

A apendicite aguda é a afecção inflamatória do apêndice vermiforme, e constitui a principal causa de abdome agudo cirúrgico nos países ocidentais ${ }^{1}$. A incidência durante a vida é estimada em $7-8 \%{ }^{2}$, acometendo sobretudo adolescentes e adultos jovens ${ }^{3}$. O diagnóstico precoce é fundamental para evitar atrasos na instituição do tratamento e a evolução para complicações potencialmente fatais, como perfuração, abscesso, peritonite e sepse.

A despeito dos recentes relatos de sucesso do tratamento não operatório com antibioticoterapia isolada ${ }^{4,5}$, a apendicectomia, seja por via aberta ou laparoscópica, é tradicionalmente o tratamento de escolha no manejo da apendicite aguda. Embora a preferência pelas técnicas minimamente invasivas seja uma tendência mundial, a cirurgia aberta ainda é a realidade na maioria dos hospitais públicos devido à ausência de capacitação da equipe e/ou da indisponibilidade de material específico para laparoscopia 6 .

O índice de complicações pós-operatórias é variável de acordo com a fase da doença e técnica cirúrgica empregada. Apesar dos bons resultados e baixa morbidade do tratamento convencional, a abordagem minimamente invasiva tem sido associada a menor incidência geral de complicações, menor tempo de internação e retorno mais precoce às atividades rotineiras? ${ }^{7}$.

O presente trabalho tem por objetivo comparar a incidência de complicações pós-operatórias entre as técnicas de apendicectomia aberta e laparoscópica para o tratamento da apendicite aguda em um hospital público de referência em emergência.

\section{MÉTODOS}

Trata-se de um estudo de coorte retrospectiva baseada em revisão de prontuários de todos os pacientes submetidos a apendicectomia por suspeita de apendicite aguda no serviço de Cirurgia Geral do
Hospital de Pronto Socorro de Porto Alegre, durante o período de quatro anos compreendido entre novembro de 2015 e novembro de 2019. Foram avaliados dados demográficos, estratificação de risco cirúrgico da Sociedade Americana de Anestesiologia (ASA), tempo de evolução dos sintomas, exames de imagem complementares, técnica cirúrgica, técnica anestésica e tipo de incisão, achados transoperatórios, necessidade de drenos ou ostomias, tempo cirúrgico, tempo de permanência hospitalar, incidência de complicações pós-operatórias, necessidade de reintervenção e experiência do cirurgião.

Todos os pacientes foram operados por residentes do serviço de Cirurgia Geral e do Trauma, sob supervisão dos cirurgiões preceptores e plantonistas da instituição. A técnica operatória foi definida a critério do cirurgião responsável e também de acordo com a disponibilidade de material para o procedimento. A fase evolutiva da apendicite foi determinada de acordo com o aspecto transoperatório e confirmada por estudo anatomopatológico, sendo classificada em não complicada (apêndice de aspecto normal; com hiperemia e edema; ou com exsudato fibrinoso) ou complicada (apêndice com necrose e abscesso; ou supurado). As complicações pós-operatórias foram estratificadas de acordo com a classificação de Clavien-Dindo ${ }^{8}$.

Os dados coletados foram registrados e analisados em banco de dados secundário no software IBM SPSS ${ }^{\circledR}$. As variáveis contínuas foram descritas através de médias e desvios-padrão ou medianas e intervalo interquartil, e as categóricas através de tabelas de frequência por meio de valores absolutos e proporções. A amostra foi dividida em dois grupos de estudo (cirurgia aberta e laparoscópica), de acordo com a técnica utilizada. $O$ teste $t$ de Student para amostras independentes foi utilizado para análise de variáveis contínuas com distribuição normal e os testes Chi-quadrado e exato de Fisher foram aplicados para variáveis categóricas. Em todos os testes foi estabelecido um intervalo de confiança de 95\% (IC = 95\%) e grau de significância estatística de 
$5 \%(p<0,05)$. O trabalho foi submetido e aprovado pelo Comitê de Ética em Pesquisa do Hospital de Pronto Socorro de Porto Alegre.

\section{RESULTADOS}

No período analisado, 358 pacientes foram submetidos a apendicectomia por suspeita de apendicite aguda na instituição. A idade média foi de $32 \pm 13$,8 anos, com predomínio do sexo masculino $(58,9 \% ; n=211)$. Em relação a comorbidades, $97,7 \%$ da amostra apresentava baixo risco cirúrgico (ASA I ou II). Duzentos e oito indivíduos $(58,1 \%)$ foram submetidos à cirurgia aberta, $150(41,9 \%)$ à cirurgia laparoscópica, e em $8 \%$ dos casos foi necessária conversão para cirurgia aberta ou laparotomia. As características da amostra são apresentadas em detalhe na Tabela 1.

Tabela 1: Características basais e operatórias.

\begin{tabular}{lc}
\hline Características basais e operatórias & \\
\hline Gênero $(\%)$ & $211(58,9)$ \\
Masculino & $147(41,1)$ \\
Feminino & $32 \pm 13,8(10-78)$ \\
\hline Idade (anos) & $2,2 \pm 2,1(1-20)$ \\
\hline Tempo de evolução (dias) & $273(76,6)$ \\
\hline ASA (\%) & $77(21,5)$ \\
I & $8(2,2)$ \\
II & \\
III & $2(0,6)$ \\
\hline Diagnóstico (\%) & $147(41,1)$ \\
\hline Exame clínico & $208(58,1)$ \\
Ultrassonografia transabdominal & $1(0,3)$ \\
Tomografia Computadorizada & \\
Ressonância Nuclear Magnética & \\
\hline Técnica anestésica (\%) & $314(87,7)$ \\
Geral & $44(12,2)$ \\
Raquianestesia & Continua...
\end{tabular}

Tabela 1: Continuação

\begin{tabular}{lc}
\hline Técnica operatória (\%) & \\
Aberta & $208(58,1)$ \\
Davis & $196(54,7)$ \\
Mediana & $12(3,4)$ \\
Laparoscópica & $138(38,5)$ \\
Convertida & $12(3,4)$ \\
\hline
\end{tabular}

Não foram observadas diferenças estatisticamente significantes entre os grupos (técnica aberta vs. laparoscópica) em relação a idade, classificação ASA, tempo de evolução dos sintomas e fase evolutiva da doença. $O$ gênero feminino esteve relacionado a maior indicação da cirurgia laparoscópica com significância estatística. Em relação aos achados intraoperatórios (Tabela 2), 30,4\% das apendicites foram classificadas como complicadas.

O estudo anatomopatológico diagnosticou três casos de tumores primários do apêndice, sendo dois tumores carcinoides e um adenocarcinoma mucinoso, além de um caso de verminose como etiologia da apendicite. Em cinco pacientes, o diagnóstico diferencial foi determinado no transoperatório, com três casos de doença inflamatória pélvica, um caso de tumor perfurado de sigmoide e um caso de diverticulite. O exame microscópico dos espécimes cirúrgicos, porém, revelou um total de 11 casos de apêndice cecal dentro dos limites da normalidade.

O tempo cirúrgico médio foi de $88 \pm 39$ minutos, sendo significativamente maior nas cirurgias laparoscópicas (104 vs. 79,3 minutos, respectivamente; $\mathrm{p}<0,001)$. Os residentes do primeiro ano realizaram a maioria das apendicectomias abertas $(74,2 \%)$, enquanto a técnica laparoscópica foi executada predominantemente por residentes do segundo ano $(59,6 \%)$. A duração da internação hospitalar variou de 1 a 43 dias, com média de 2,9 dias, sem diferenças em relação à técnica realizada.

Tabela 2: Comparação entre as técnicas laparoscópica e aberta em relação a variáveis perioperatórias.

\begin{tabular}{|c|c|c|c|c|}
\hline & $\begin{array}{c}\text { VLP } \\
(n=138)\end{array}$ & $\begin{array}{c}\text { Aberta } \\
(n=220)\end{array}$ & $\begin{array}{c}\text { Total } \\
(\mathrm{n}=358)\end{array}$ & p \\
\hline Idade (anos) & $\begin{array}{c}31,7 \pm 13,4 \\
(10-78)\end{array}$ & $\begin{array}{c}32,2 \pm 14 \\
(10-78)\end{array}$ & $\begin{array}{c}32 \pm 13,8 \\
(10-78)\end{array}$ & ns \\
\hline \multicolumn{5}{|l|}{ Gênero (\%) } \\
\hline Masculino & $67(48,6)$ & $144(65,5)$ & $211(58,9)$ & 0,001 \\
\hline Feminino & $71(51,4)$ & $76(34,5)$ & $147(41,1)$ & \\
\hline \multicolumn{5}{|l|}{ ASA (\%) } \\
\hline I & $108(78,3)$ & $165(75)$ & $273(76,2)$ & \\
\hline II & $29(21)$ & $48(21,8)$ & $77(21,5)$ & ns \\
\hline III & $1(0,7)$ & $7(3,2)$ & $8(2,3)$ & \\
\hline \multicolumn{5}{|l|}{ Apendicite (\%) } \\
\hline Não complicada & $93(67,4)$ & $156(71)$ & $249(69,6)$ & ns \\
\hline Complicada & $45(32,6)$ & $64(29,1)$ & $109(30,4)$ & \\
\hline Ostomia (\%) & 0 & $2(1)$ & $2(0,6)$ & ns \\
\hline Dreno abdominal (\%) & $11(8)$ & $5(2,3)$ & $16(4,5)$ & 0,016 \\
\hline
\end{tabular}


Tabela 2: Continuação.

\begin{tabular}{|c|c|c|c|c|}
\hline Tempo cirúrgico (min) & $\begin{array}{c}104 \pm 35,2 \\
(35-210)\end{array}$ & $\begin{array}{c}79,3 \pm 38,8 \\
(30-250)\end{array}$ & $\begin{array}{c}88 \pm 39 \\
(30-250) \\
\end{array}$ & 0,001 \\
\hline Tempo de internação (dias) & $\begin{array}{c}2,8 \pm 3,3 \\
(1-22)\end{array}$ & $\begin{array}{c}3 \pm 4 \\
(1-43)\end{array}$ & $\begin{array}{c}2,9 \pm 3,7 \\
(1-43)\end{array}$ & ns \\
\hline Mortalidade (\%) & 0 & $1(0,5)$ & $1(0,3)$ & ns \\
\hline \multicolumn{5}{|l|}{ Anatomopatológico (\%) } \\
\hline Apendicite aguda & $134(97,1)$ & $210(95,5)$ & $344(96,1)$ & \multirow{3}{*}{ ns } \\
\hline Branca & $3(2,2)$ & $8(3,6)$ & $11(3,1)$ & \\
\hline Neoplasia de apêndice & $1(0,7)$ & $2(0,9)$ & $3(0,8)$ & \\
\hline \multicolumn{5}{|l|}{ Experiência do cirurgião (\%) } \\
\hline R1 & $26(19,1)$ & $161(74,2)$ & $187(53)$ & \multirow{3}{*}{0,001} \\
\hline R2 & $81(59,6)$ & $39(18)$ & $120(34)$ & \\
\hline R3 & $29(21,3)$ & $17(7,8)$ & $46(13)$ & \\
\hline
\end{tabular}

*ns: estatisticamente não significante.

A descrição das complicações pós-operatórias e a classificação de Clavien-Dindo é apresentada em detalhe na Tabela 3. A maioria das intercorrências $(80,2 \%)$ foi definida como menor (grau I e II) e, portanto, tratadas apenas com medidas de suporte e tratamento farmacológico, sem necessidade de reintervenção. A taxa de complicações pós-operatórias foi de $21,2 \%$ ( $n=76)$, e a infecção de ferida operatória, íleo adinâmico e abscesso intra-abdominal foram as intercorrências mais comuns. Os eventos de sítio cirúrgico, incluindo infecção de ferida operatória, bem como o íleo, foram significativamente mais frequentes na cirurgia aberta. A ocorrência de fístulas, bridas e hérnias incisionais foram mais observadas na técnica aberta, embora sem relevância estatística. Em 3,9\% dos casos $(n=14)$, foi necessária uma reintervenção cirúrgica para a abordagem de complicações. Um caso de óbito intra-hospitalar foi reportado, referente a uma paciente do sexo feminino de 78 anos de idade, cirrótica, submetida a apendicectomia aberta através de laparotomia mediana por apendicite complicada com abscesso retroperitoneal extenso.

Tabela 3: Análise comparativa das complicações pós-operatórias entre as técnicas laparoscópica e aberta.

\begin{tabular}{|c|c|c|c|c|c|c|c|}
\hline \multirow[b]{2}{*}{ Complicações } & \multicolumn{2}{|c|}{$\begin{array}{c}\text { VLP } \\
(n=138)\end{array}$} & \multicolumn{2}{|c|}{$\begin{array}{c}\text { Aberta } \\
(n=220)\end{array}$} & \multicolumn{2}{|c|}{$\begin{array}{c}\text { Total } \\
(\mathrm{n}=358)\end{array}$} & \multirow[t]{2}{*}{$\mathbf{p}$} \\
\hline & $\mathbf{n}$ & $\%$ & $\mathbf{n}$ & $\%$ & $\mathbf{N}$ & $\%$ & \\
\hline Infecção de ferida operatória & 3 & 2,2 & 25 & 11,4 & 28 & 7,8 & 0,001 \\
\hline Eventos de sítio cirúrgico & 2 & 1,4 & 16 & 7,3 & 18 & 5 & 0,013 \\
\hline Íleo adinâmico & 4 & 2,9 & 20 & 9,1 & 24 & 6,7 & 0,028 \\
\hline Abscesso intra-abdominal & 12 & 8,7 & 13 & 5,9 & 25 & 7 & ns \\
\hline Suboclusão por aderências & 0 & 0 & 3 & 1,4 & 3 & 0,8 & ns \\
\hline Deiscência / fístula & 0 & 0 & 3 & 1,4 & 3 & 0,8 & ns \\
\hline Hérnia incisional & 1 & 0,7 & 5 & 2,3 & 6 & 1,7 & ns \\
\hline Choque séptico de foco abdominal & 0 & 0 & 3 & 1,4 & 3 & 0,8 & ns \\
\hline Quiloperitônio & 0 & 0 & 1 & 0,5 & 1 & 0,3 & ns \\
\hline Total & 18 & 13 & 58 & 26,4 & 76 & 21,2 & 0,003 \\
\hline Reintervenção & 7 & 5,1 & 7 & 3,2 & 14 & 3,9 & ns \\
\hline Clavien-Dindo & $\mathbf{n}$ & $\%$ & $\mathbf{n}$ & $\%$ & $\mathbf{N}$ & $\%$ & 0,013 \\
\hline 1 & 3 & 2,2 & 18 & 8,2 & 21 & 5,9 & \\
\hline II & 10 & 7,2 & 30 & 13,6 & 40 & 11,2 & \\
\hline III & 5 & 3,6 & 6 & 2,7 & 11 & 3,1 & \\
\hline IV & 0 & 0 & 3 & 1,4 & 3 & 0,8 & \\
\hline V & 0 & 0 & 1 & 0,5 & 1 & 0,3 & \\
\hline
\end{tabular}

*ns: estatisticamente não significante.

A Figura 1 ilustra a proporção entre as técnicas de apendicectomias distribuídas por ano, ao longo do período estudado. Observa-se uma tendência crescente de preferência pela cirurgia laparoscópica no serviço. 


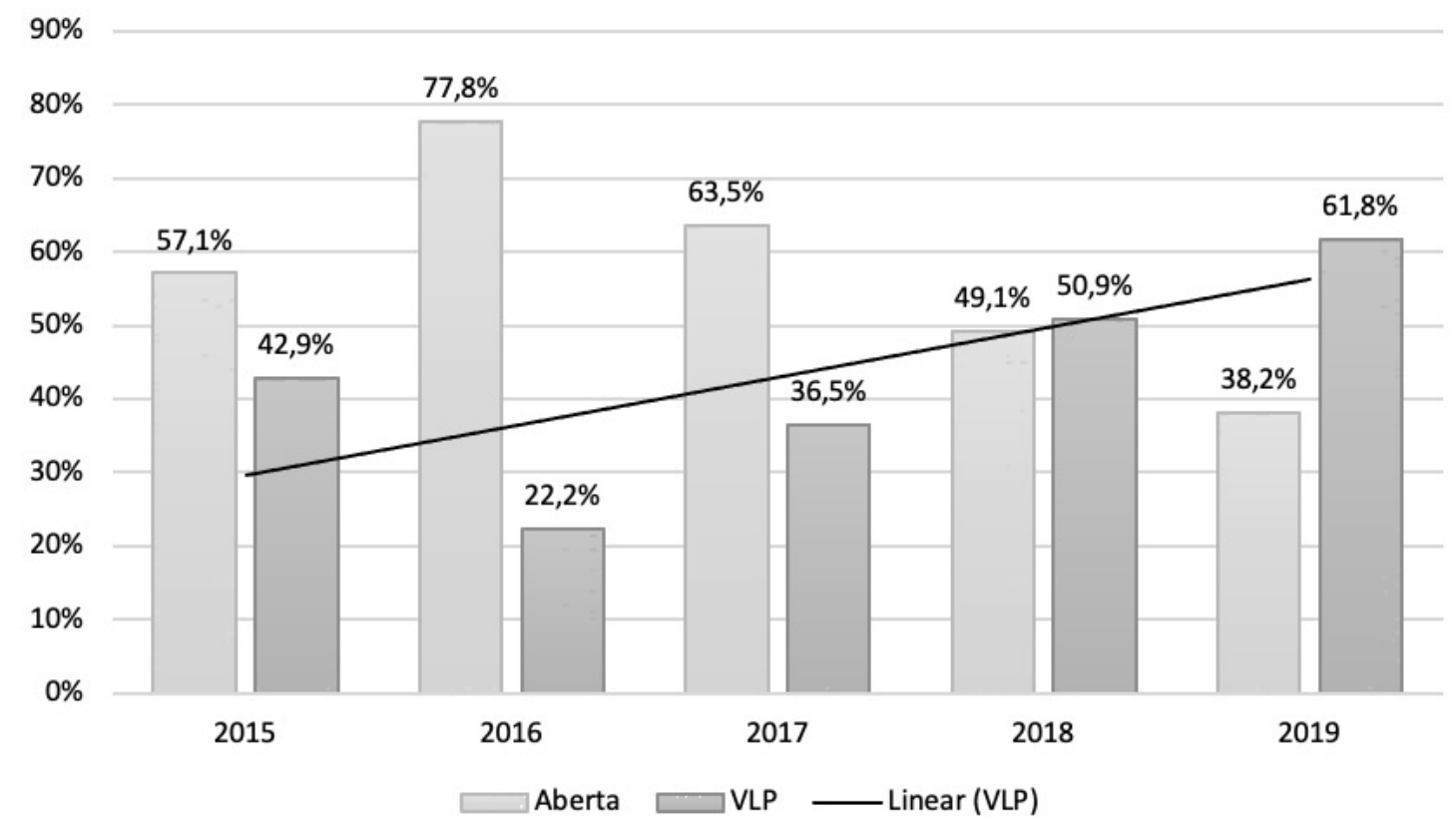

Figura 1: Distribuição das técnicas cirúrgicas por ano.

\section{DISCUSSÃO}

A apendicectomia clássica, inicialmente descrita por Charles McBurney em 1893, foi o tratamento de escolha para apendicite aguda por mais de um século, até a primeira descrição da técnica laparoscópica por Kurt Semm, em $1983^{9}$. Na tentativa de definir os possíveis benefícios da abordagem minimamente invasiva no tratamento de uma doença que, historicamente, tem apresentado bons resultados e baixa morbimortalidade com a cirurgia convencional ${ }^{10}$, diversos estudos têm sido conduzidos com objetivo de comparar as técnicas.

Revisões sistemáticas e metanálises recentes ${ }^{9,11,12}$ demonstraram que a apendicectomia laparoscópica é tão segura e eficaz quanto a cirurgia aberta, com potenciais benefícios em termos de melhor controle da dor no pós-operatório, menor incidência de complicações, menor risco de hérnia incisional, alta hospitalar com retorno às atividades habituais mais precoce e melhor resultado cosmético. Apresenta, ainda, a vantagem técnica de facilitar o acesso a toda cavidade abdominal, permitindo o diagnóstico diferencial em casos duvidosos e a exploração com lavagem completa dos espaços peritoneais em casos complicados. Por outro lado, resultados conflitantes em relação a um possível maior risco de sangramento intraoperatório, formação de abscessos intra-abdominais, infecções do trato urinário, bem como tempo cirúrgico mais prolongado e custos associados foram obstáculos à adoção da laparoscopia como técnica preferencial na última década ${ }^{13,14}$.
A metanálise cumulativa conduzida por Ukai et al. ${ }^{15}$, que incluiu 64 ensaios clínicos randomizados publicados entre 1992 a 2013, identificou mudanças significativas na evidência científica disponível durante o período estudado, com progressiva tendência a menor disparidade entre as técnicas em relação às variáveis abscesso intra-abdominal e tempo cirúrgico. Esses achados podem ser resultado da maior experiência dos cirurgiões e otimização da técnica laparoscópica adquirida ao longo dos anos. Em consonância com esses resultados, as atuais diretrizes da European Association of Endoscopic Surgery (EAES/2015) $)^{16}$ e da World Society of Emergency Surgery (WSES/2016)17, recomendam a apendicectomia laparoscópica como procedimento de escolha para tratamento da apendicite não complicada e complicada, inclusive em populações especiais como crianças, idosos, gestantes e obesos, dada a disponibilidade técnica e de material.

No Brasil, o número de apendicectomias laparoscópicas cresceu $279,7 \%$ entre os anos de 2008 e 2014 no serviço público, sendo que 57\% desses procedimentos foram executados na região $\mathrm{Sul}^{6}$. Apesar do importante e progressivo crescimento da laparoscopia observado na última década, com destaque para nossa região, a maioria das apendicectomias nos pacientes atendidos em hospitais públicos brasileiros ainda é realizada por via aberta ${ }^{6}$. A casuística apresentada reflete a realidade de um hospital público referência em emergência, que enfrenta constantes entraves à realização da laparoscopia em decorrência de falhas ou indisponibilidade de material, ausência de capacitação técnica de todos cirurgiões plantonistas, além do comprometimento com 
o ensino de residentes, que necessitam treinamento nas duas vias de acesso cirúrgico.

Em nossa série, verificou-se perfil de pacientes predominantemente masculino, de adultos jovens com baixo risco cirúrgico e tempo de evolução dos sintomas relativamente prolongado. Cerca de um terço das apendicites foi classificado como complicada. Pouco mais da metade dos pacientes foi submetida à apendicectomia aberta, embora uma tendência de aumento na proporção de laparoscopias seja observada nos últimos dois anos (Figura 1). Houve uma maior indicação da apendicectomia laparoscópica no sexo feminino, em concordância com diversos autores ${ }^{18}$ que sinalizam o papel da técnica no diagnóstico diferencial de doenças anexiais, sobretudo em mulheres em idade reprodutiva.

O tempo cirúrgico médio foi superior na técnica laparoscópica em 24,7 minutos ( $p<0,001$ ), o que pode ser justificado pela curva de aprendizado dos residentes e tempo de montagem e preparação do equipamento de laparoscopia. Essa diferença está em conformidade com os resultados das metanálises conduzidas por Li et al. ${ }^{11} \mathrm{e}$ Wei et al. ${ }^{12}$, que demonstraram diferenças de 15,1 e 10,7 minutos entre as técnicas, respectivamente. É interessante notar que, recentemente, essa discrepância em termos de tempo cirúrgico vem diminuindo, com relatos de diferenças de apenas 8,6 minutos $^{11}$, sendo irrelevantes na prática.

A taxa de conversão para cirurgia aberta em nossa amostra foi de $8 \%$, majoritariamente por dificuldades técnicas, valor muito semelhante ao reportado por outro estudo gaúcho $(8,4 \%)^{19}$ e por metanálise de ensaios clínicos randomizados $(9,5 \%)^{11}$. O índice geral de complicações foi de $21,2 \%$, em conformidade com a literatura (3-28,7\%) ${ }^{16}$, sendo significativamente menor na técnica laparoscópica em relação a aberta (13\% vs. $26,4 \%$, respectivamente; $p<0,003$ ). Essa diferença deveu-se, sobretudo, à maior incidência de infecções de ferida operatória na cirurgia aberta (11,4\% vs. 2,2\%; $p=0,001)$. Da mesma forma, recente revisão publicada pela Cochrane ${ }^{9}$ evidenciou que a probabilidade de infecções de sítio cirúrgico nos pacientes submetidos a laparoscopia é aproximadamente a metade do risco daqueles operados por via aberta. Outras complicações de ferida operatória, como seromas e hematomas, bem como o íleo adinâmico, também foram significativamente mais frequentes na técnica aberta. Os abscessos intraabdominais foram mais observados na laparoscopia, embora sem significância estatística. A necessidade de reintervenção foi semelhante entre as técnicas.

O índice de apendicectomias negativas foi de 3,9\% sem estratificação por gênero, em conformidade com as séries mais recentes na literatura $(6-8 \%)^{20}$ e com outro estudo gaúcho $(6,3 \%)^{19}$. A taxa de mortalidade pós-operatória na literatura é estimada em 0,07-0,7\% nos pacientes sem perfuração do apêndice vermiforme e $0,5-2,4 \%$ em casos de apendicite supurada ${ }^{21}$. Em nossa série, uma paciente $(0,2 \%)$, idosa e com comorbidades graves, foi a óbito em decorrência de uma apendicite rota com quadro sistêmico importante.

O tempo de internação não apresentou diferença estatisticamente significante em relação às técnicas estudadas; no entanto, diversos estudos na literatura demonstram uma menor permanência hospitalar dos pacientes submetidos à laparoscopia ${ }^{9,11,14}$. Os critérios e rotinas para alta hospitalar adotadas em nosso serviço podem contribuir para esse achado. Dada a ausência de um instrumento para avaliação objetiva da dor pós-operatória e da satisfação do paciente, essas variáveis não foram objeto do presente estudo.

Entre as limitações do trabalho, destaca-se a sua natureza retrospectiva e não randomizada, bem como aquelas inerentes à utilização de registros de prontuários como fonte de dados. A escolha da técnica foi baseada na experiência e preferência do cirurgião, considerando ainda o estado clínico do paciente, o que pode representar um viés de seleção.

A presente série de casos confirma a aplicabilidade da laparoscopia para o tratamento da apendicite aguda no contexto de um hospital público e de ensino, a despeito dos desafios enfrentados para sua execução. Ademais, demonstrou benefícios da técnica em relação à evolução pós-operatória, de forma que os autores recomendam sua indicação rotineira nos serviços que dispõem de material e capacitação técnica da equipe cirúrgica.

\section{REFERÊNCIAS}

1. Ferris M, Quan S, Kaplan BS, Molodecky N, Ball CG, Chernoff $\mathrm{GW}$, et al. The global incidence of appendicitis: a systematic review of population-based studies. Ann Surg. 2017;266(2):237-41.

2. Alvarado A. Improved Alvarado Score (MANTRELS) for the early diagnosis of acute appendicitis. Int J Surg Res Pract. 2019;6:098.
3. Buckius MT, McGrath B, Monk J, Grim R, Bell T, Ahuja V. Changing epidemiology of acute appendicitis in the United States: study period 1993-2008. J Surg Res. 2012;175(2):185-190.

4. Hansson J, Körner U, KhorramManesh A, Solberg A, Lundholm K. Randomized clinical trial of antibiotic therapy versus appendicectomy as primary treatment of acute appendicitis in unselected patients. Br J Surg. 2009;96(5):473-81.

5. Hirano ES, Pereira BMT, BustorffSilva JM, Rizoli S, Nascimento B Jr, Fraga GP. Apendicite aguda não complicada em adultos: tratamento cirúrgico ou clínico? Rev Col Bras Cir. 2012;39(2)159-63. 
6. Santos F, Cavasana GF, Campos T. Perfil das apendicectomias realizadas no Sistema Público de Saúde do Brasil. Rev Col Bras Cir. 2017;44(1):4-8.

7. Moreira LF, Garbin HI, DaNatividade GR, Silveira BW, Xavier TV. Fatores preditores de complicações pós-operatórias em apendicectomias. Rev Col Bras Cir. 2018;45(5):e1920.

8. Moreira LF, Pessôa MC, Mattana DS, Schmitz FF, Volkweis BS,

Antoniazzi JL, et al. Adaptação cultural e teste da escala de complicações cirúrgicas de Clavien-Dindo traduzida para o Português do Brasil. Rev Col Bras Cir. 2016;43(3):141-8.

9. Jaschinski T, Mosch CG, Eikermann M, Neugebauer EA, Sauerland S. Laparoscopic versus open surgery for suspected appendicitis. Cochrane Database Syst Rev. 2018;11(11):CD001546.

10. Nahas SC, Lourenção JL, Borba MR, Marques CFS, Nahas CSR, Dias AR. Apendicectomia laparoscópica: utilizando-a como rotina. Rev Bras Videocir. 2004;2(3):118-22.

11. Li X, Zhang J, Sang L, Zhang W, Chu Z, Li X, et al. Laparoscopic versus conventional appendectomy a meta-analysis of randomized controlled trials. BMC Gastroenterol. 2010;10:129.
12. Wei B, Qi CL, Chen TF, Zheng ZH, Huang JL, Hu BG, et al. Laparoscopic versus open appendectomy for acute appendicitis: a meta-analysis. Surg Endosc. 2011;25(4):1199-208.

13. Biondi A, Di Stefano C, Ferrara F, Bellia A, Vacante M, Piazza L. Laparoscopic versus open appendectomy: a retrospective cohort study assessing outcomes and costeffectiveness. World J Emerg Surg. 2016;11(1):44

14. Athanasiou C, Lockwood S, Markides GA. Systematic review and metaanalysis of laparoscopic versus open appendicectomy in adults with complicated appendicitis: an update of the literature. World $J$ Surg. 2017;41(12):3083-99.

15. Ukai T, Shikata S, Takeda H, Dawes L, Noguchi Y, Nakayama T, et al. Evidence of surgical outcomes fluctuates over time: results from a cumulative meta-analysis of laparoscopic versus open appendectomy for acute appendicitis. BMC Gastroenterol. 2016;16:37.

16. Gorter RR, Eker HH, GorterStam MA, Abis GS, Acharya A, Ankersmit M, et al. Diagnosis and management of acute appendicitis. EAES consensus development conference 2015. Surg Endosc. 2016;30(11):4668-90.
17. Di Saverio S, Birindelli A, Kelly MD, Catena F, Weber DG, Sartelli M, et al. WSES Jerusalem guidelines for diagnosis and treatment of acute appendicitis. World J Emerg Surg. 2016;11:34.

18. Sauerland S, Jaschinski T, Neugebauer EA. Laparoscopic versus open surgery for suspected appendicitis. Cochrane Database Syst Rev. 2010,10:CD001546.

19. Navarini D, Valiati AA, Rodrigues RR, Aita LN, Migliavaca A, Guimarães JR. Apendicectomia laparoscópica versus aberta: análise retrospectiva. Rev HCPA. 2009;29(2):115-9.

20. Mariadason JG, Wang WN, Wallack MK, Belmonte A, Matari $\mathrm{H}$. Negative appendicectomy rate as a quality metric in the management of appendicitis: impact of computed tomography, Alvarado score and the definition of negative appendicectomy. Ann R Coll Surg Engl. 2012;94(6):395-401.

21. Sartelli M, Baiocchi GL, Di Saverio S, Ferrara F, Labricciosa FM, Ansaloni L, et al. Prospective observational study on acute appendicitis worldwide. World J Emerg Surg. 2018;13(1):19.

Recebido: 05 Nov, 2020 Aceito: 09 Jun, 2021 